\title{
EFECTO DE DOS LEGUMINOSAS Y BANANO MADURO EN LA PRODUCCIÓN Y REPRODUCCIÓN DE CONEJOS NUEVA ZELANDA
}

\section{EFFECT OF TWO LEGUMES AND RIPE BANANAS AT THE PRODUCTION AND REPRODUCTION OF NEW ZEALAND RABBITS}

\author{
${ }^{\bullet}$ Adolfo Sánchez Laiño ${ }^{1,2}$, Emma Torres Navarrete ${ }^{1,2}$, Gary Meza Bone ${ }^{1,2}$, Kléber Estupiñán Véliz², \\ Yenny Torres Navarrete ${ }^{3}$, Alexandra Barrera Álvarez ${ }^{1,2}$, Yessica Mackencie Álvarez ${ }^{4}$, Linda López Intriago ${ }^{1,2}$ \\ ${ }^{I}$ Dirección de Investigación Científica y Tecnológica, Universidad Técnica Estatal de Quevedo. Campus finca experimental \\ "La María”, km 7 vía Quevedo-El Empalme. C. P. 73. Mocache, Los Ríos, Ecuador. ${ }^{\circ}$ adolsanlai@hotmail.com \\ ${ }^{2}$ Facultad de Ciencias Pecuarias, Universidad Técnica Estatal de Quevedo. Campus finca experimental "La María", km 7 vía \\ Quevedo-El Empalme. C. P. 73. Mocache, Los Ríos, Ecuador. \\ ${ }^{3}$ Facultad de Ciencias Agrarias, Universidad Técnica Estatal de Quevedo. Campus Ing. Manuel Haz Álvarez, $\mathrm{km} 1.5$ vía a \\ Santo Domingo de los Tsáchilas. C. P. 73. Quevedo, Los Ríos, Ecuador. \\ ${ }^{4}$ Unidad de Estudios a Distancia, Universidad Técnica Estatal de Quevedo. Campus Ing. Manuel Haz Álvarez, $\mathrm{km} 1.5$ vía a \\ Santo Domingo de los Tsáchilas. C. P. 73. Quevedo, Los Ríos, Ecuador.
}

\section{RESUMEN}

$\mathrm{L}^{\mathrm{a}}$ a investigación tuvo los siguientes objetivos: a) Determinar la leguminosa tropical (Pueraria phaseoloides (Roxb) Benth y Gliricidia sepium) y el nivel de banano maduro (BM) (50, 75, 100 y 100, 125, $150 \mathrm{~g}$ animal $^{-1}$ día $^{-1}$ ), que permita incrementar los parámetros reproductivos (fase uno) y productivos (fase dos), en conejos Nueva Zelanda. b) Determinar la Relación beneficio/costo de los tratamientos. Se utilizó 28 y 42 conejos respectivamente. Se aplicó un arreglo factorial $2 \times 3+1$, en un diseño de bloques completamente al azar (DBCA), con cuatro y tres repeticiones. Se utilizó la prueba de Tukey $(\mathrm{p} \leq 0.05)$. El mayor consumo fue para el kudzu $(\mathrm{p}<0.01)$ durante las fases 1 y 2 (79.97 y 43.26 g MS animal ${ }^{-1}$ día $\left.^{-1}\right)$, incrementando $(\mathrm{p}<0.01)$ el peso y tamaño de la camada al destete $(2,165.0$ g y 5.33). El mayor consumo de banano maduro $(B M)(p<0.01)$ lo registró el nivel 3, durante las fases 1 y 2 (38.39 y 31.39 g MS animal ${ }^{-1}$ día $\left.^{-1}\right)$. El consumo de leguminosas y BM no afectó $(p>0.05)$ el peso de las hembras al parto, el periodo de gestación, peso y tamaño de la camada al nacimiento. Los niveles de BM no influenciaron $(\mathrm{p}>0.05)$ sobre la ganancia de peso, índice de conversión alimenticia (ICA), peso final (PF), peso a la canal (PC) y rendimiento a la canal ( $\mathrm{RC})$. Los tratamientos en base a leguminosas tropicales y niveles de BM superaron al testigo $(p<0.01)$. La mayor rentabilidad en la fase 1 y 2 se obtuvo al combinar kudzu tropical más 100 y 75 g y 125 y $100 \mathrm{~g}_{\text {de BM }}$ animal-1 día ${ }^{-1}$ (30.34 y 29.48\%).

Palabras clave: Reproducción, producción, conejos, alimentación, leguminosas, banano.

Recibido: 20-Septiembre-2012. Recibido en forma corregida: 28-Noviembre-2012 Aceptado: 13-Diciembre-2012.

Publicado como ARTíCULO CIENTÍFICO en Ciencia y Tecnología 5(2): 27-31. 2012
$\mathrm{T}$ his study was conducted to evaluate the following objectives: a) Determining the tropical leguminous plant (Pueraria phaseoloides (Roxb) Benth and Gliricidia sepium) and the level of ripe bananas (RB) $\left(50,75,100,100,125,150 \mathrm{~g}^{\text {animal }}{ }^{-1}\right.$ day $\left.^{-1}\right)$, as for increasing the reproductive parameters (phase one) and productive (phase two), in New Zealand rabbits. b) Determining the relation benefit / cost of treatments. They were used 28 and 42 rabbits respectively. It was applied a factorial arrangement $2 \times 3+1$, at random completely blocks design (RCBD) with four and three replications. It was used the Tukey test $(p \leq 0.05)$. The greatest consumption was for kudzu $(p<0.01)$ during phases 1 and 2 (79.97 and $43.26 \mathrm{~g}$ dry matter (DM) animal ${ }^{-1}$ day $\left.^{-1}\right)$, it increased $(p<0.01)$ weight and litter size at weaning $(2165.0 \mathrm{~g} \mathrm{5.33})$. The greatest consumption of RB $(p<0.01)$ was registered on level 3 , during phases 1 and 2 (38.39 and $31.39 \mathrm{~g} \mathrm{DM}$ animal $^{-1}$ day $\left.^{-1}\right)$. The consumption of leguminous plant and RB did not affect ( $p>0.05)$ the weight of females at birth, the gestation period, weight and litter size at birth. RB levels did not influence ( $>0.05)$ ADG average dairy gain weight gain, feed conversion ratio (FCR), final weight (FW), body weight carcass (BWC) and carcass yield (RC). Treatments based on tropical legumes and RB levels exceeded the control $(p<0.01)$. The greatest profitability in phase 1 and 2 was obtained by combining tropical kudzu 100 and $75125 \mathrm{~g}$ and $100 \mathrm{~g} \mathrm{BM}$ animal $^{-1}$ day $^{-1}$ (30.34 and 29.48\%).

Key words: Reproduction, production, rabbits, food, leguminous plant, ripe bananas. 


\section{INTRODUCCIÓN}

$\mathrm{L}$ a alimentación del conejo, como el de las otras especies de interés zootécnico, representa el rubro más elevado dentro de los costos de producción, siendo necesaria la búsqueda de nuevas fuentes alimenticias tendientes a reducirlo, sin descuidar los requerimientos nutricionales del animal en cada una de sus fases biológicas (gestación, lactancia, cría, recría). En el orden de desarrollar programas alimenticios para conejos de manera más eficiente utilizando forrajes tropicales, es necesario tener conocimiento de sus cualidades específicas para esta especie, incluyendo digestibilidad, palatabilidad, contenido nutricional y los efectos sobre el funcionamiento intestinal y las enfermedades entéricas (Cheeke, 1992). Asimismo existe una limitante de información que incluya la respuesta de conejos en crecimiento a diferentes fuentes suplementarias tropicales, sus combinaciones y su impacto económico (Mbanya et al., 2005).

Dentro del ambiente tropical, existen muchas alternativas alimenticias que pueden contribuir considerablemente a aumentar la cantidad de nutrientes disponibles, siendo las leguminosas tropicales (Pueraria phaseoloides y Gliricidia sepium) y el banano (Musa paradisíaca) ejemplos de este potencial. Aunque el banano se cultiva principalmente para la exportación y para el consumo doméstico de la población humana, hay grandes cantidades disponibles de esta fruta que pueden emplearse en la alimentación animal. El banano de rechazo constituye una buena fuente de carbohidratos para los conejos.

\section{Materiales y MÉTODOS}

$\mathrm{L}$ a investigación se ejecutó en el Programa de Especies Menores del campus finca experimental "La María" propiedad de la Universidad Técnica Estatal de Quevedo (UTEQ), localizado en el km 7 de la vía Quevedo - Mocache, provincia de Los Ríos. Situada entre las coordenadas geográficas $70^{\circ} 27^{\prime} 13^{\prime}$ ' de latitud Sur y $01^{\circ} 06$ '02" de longitud Oeste, a una altura de 73 $\mathrm{msnm}$, con una precipitación anual de 2,286.6 $\mathrm{mm}^{2}$ año ${ }^{-1}$, temperatura promedio anual de $25^{\circ} \mathrm{C}$, humedad relativa de $84.0 \%$, heliofanía media anual de 974.0 horas luz y topografía irregular.

En la fase de reproducción se aplicó un arreglo factorial 2 (leguminosas) x 3 (niveles de banano maduro) + 1 (testigo), dentro de un DBCA (se bloqueó el peso inicial de las unidades experimentales), con cuatro repeticiones, igual arreglo y diseño experimental se utilizó en la fase de engorde, pero con tres repeticiones. Se aplicó la prueba de Tukey $(\mathrm{p} \leq 0.05)$, y para la valoración económica la Relación Beneficio-Costo.
En la primera etapa se utilizaron 28 conejas Nueva Zelanda de cinco meses de edad con un peso promedio de $3,087 \pm 0,324 \mathrm{~kg}$, en la segunda se utilizaron 42 gazapos de 35 días de edad. Los factores bajo estudio fueron: Reproducción: a) Leguminosas: kudzu tropical (Pueraria phaseoloides) y Matarratón (Gliricidia sepium), ad libitum y b) Niveles de banano maduro (50, 75 y $100 \mathrm{~g}$ animal $^{-1}$ día $\left.^{-1}\right)$. Producción: a) Leguminosas :kudzu tropical (Pueraria phaseoloides) y Matarratón (Gliricidia sepium), ad libitum y b) Niveles de banano maduro (100, 125 y $150 \mathrm{~g}$ animal $^{-1}$ día $\left.^{-1}\right)$.

En la reproducción las variables evaluadas fueron: Peso a la monta, peso post parto, peso y tamaño de la camada al nacimiento, peso y tamaño de la camada al destete, peso de la madre al destete, mortalidad, consumo de leguminosas y consumo de banano maduro. Mientras que en el engorde se evaluó el consumo de leguminosas, consumo de banano, consumo de alimento total, ganancia de peso, conversión alimenticia, peso y rendimiento a la canal.

\section{Resultados Y DISCUSIÓN}

Fase de gestación. El mayor $(\mathrm{p}<0.01)$ consumo de forraje, alimento total, peso y tamaño de la camada al destete lo registró el tratamiento en base a kudzu tropical (107.93 y $207.25 \mathrm{~g}$ animal $^{-1}$ día $^{-1}, 2,164.58$ g y 5.33 con un peso vivo (PV) de $406.11 \mathrm{~g}^{\text {animal }^{-1}}$ y una supervivencia durante la etapa de lactancia del $86.39 \%$ ). El consumo de forraje fue similar al reportado por García et al. (2009) (104 g MS de morera d $\mathrm{d}^{-1}$ ). El PV y la supervivencia contrastan con los reportados por López et al. (2011) (694 g animal-1 y 84.40\%), valores obtenidos practicando un destete a los 45 días de edad. El mayor consumo de kudzu, puede deberse a que los animales prefieren ingerir cantidades elevadas de leguminosas, corroborando lo indicado por Tuárez (1977); quien expresa que las leguminosas forrajeras no solo contribuyen a mejorar la producción y valor nutritivo de los forrajes, sino que también mejoran las condiciones de consumo voluntario por parte de los animales.

El mayor $(\mathrm{p}<0.01)$ consumo de banano maduro lo registró el nivel tres $\left(38.38 \mathrm{~g}_{\text {animal }}{ }^{-1} \mathrm{dí}^{-1}\right)$, la interacción "Leguminosas x banano maduro" fue significativa. Sin embargo estos no influenciaron $(\mathrm{p}>0.05)$ sobre el peso al parto, días de gestación, peso y tamaño de la camada al nacimiento. El mayor consumo de banano maduro puede deberse a la palatabilidad de esta fruta y al alto contenido de energía que posee. El consumo de banano reportado en la presente investigación supera a los de Lebas et al. (1997); Maertens (1998) y Nouel et al. (2003) (25.04 $\mathrm{g} \mathrm{animal}^{-1}$ día $\left.^{-1}\right)$. El peso al destete (350 g) es inferior a los reportados por De Blass (1989), quien 
Efecto de dos leguminosas y banano maduro en la producción y reproducción de conejos nueva zelanda

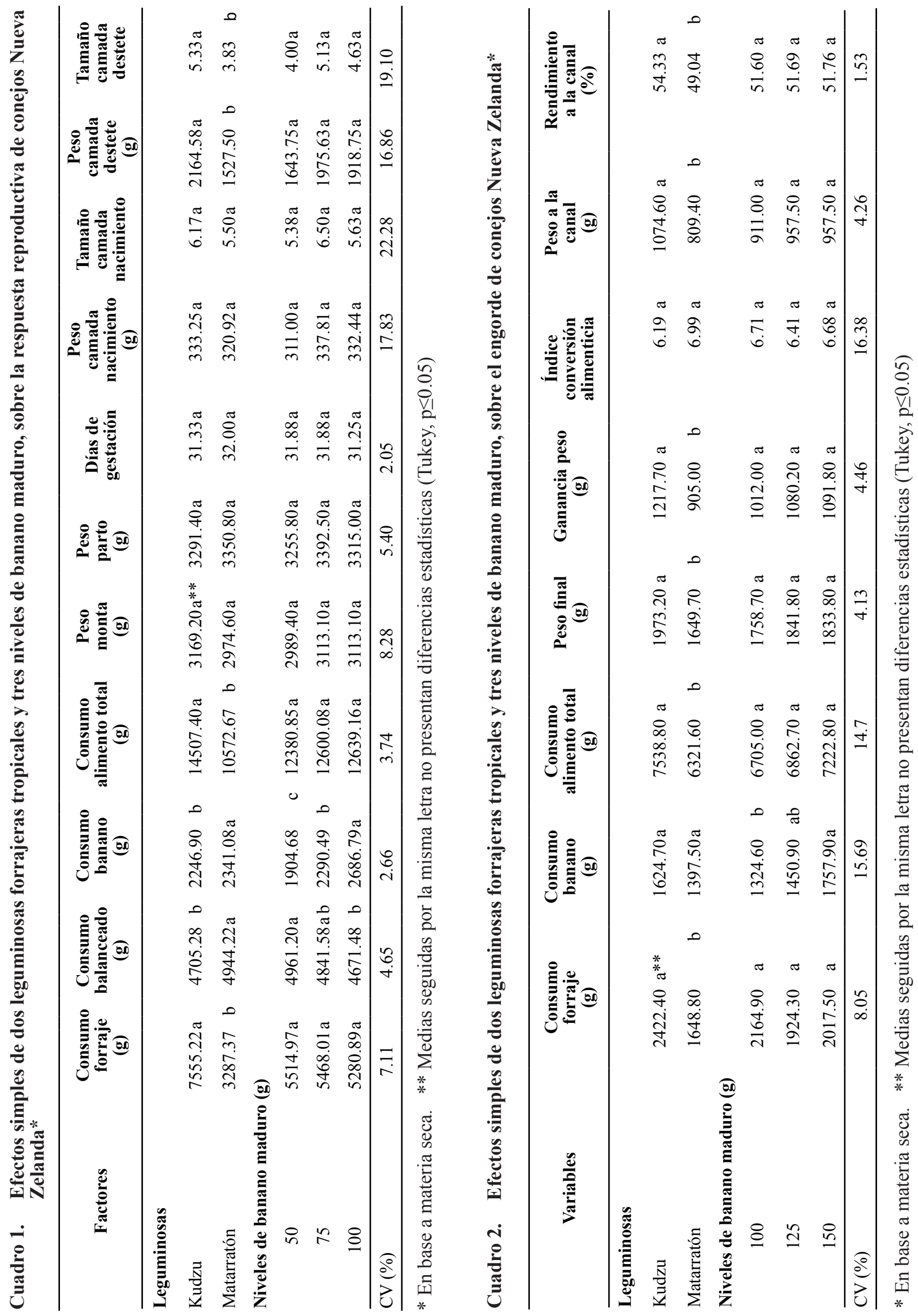


sostiene que, se debe suministrar una ración adecuada a las hembras reproductoras para conseguir gazapos de $70 \mathrm{~g}$ al nacimiento, sanos y vigorosos y de $400 \mathrm{~g}$ a la tercera o cuarta semana de vida (Cuadro 1).

Fase de engorde. El mayor $(\mathrm{p}<0.01)$ consumo de forraje, banano maduro, alimento total, ganancia de peso, peso y rendimiento a la canal (43.26, 29.01, $134.46,21.74$ y $1,074.6$ g y $54.33 \%$, respectivamente) lo registró el tratamiento en base a kudzu tropical. Los niveles de banano maduro no incrementaron $(\mathrm{p}>0.05)$ el consumo de forraje, consumo de alimento total, ganancia de peso, conversión alimenticia, peso final y rendimiento a la canal. Sin embargo en la medida en que se incrementaron los niveles de banano, el consumo del mismo se incrementó (26.98 $\left.\mathrm{g}_{\text {animal }}{ }^{-1} \mathrm{dí}^{-1}\right)$. La mayor rentabilidad se la obtuvo al combinar kudzu tropical más 125 y $100 \mathrm{~g}$ animal $^{-1}$ día $^{-1}$ de banano maduro (30.34 y $29.48 \%$ ), (Cuadros 2 y 3 ).

Estos resultados difieren con los de Nieves et al. (2009), quienes al evaluar la inclusión de follaje de leucaena (Leucaena leucocephala), naranjillo
(Trichancheera gigantea) y morera (Morus alba) en proporciones del 10, 20 y $30 \%$ en dietas balanceadas granuladas para conejos, reportaron ganancias de pesos y conversión alimenticias más eficientes en los animales que recibieron follaje de leucaena y morera (29.49 y $26.00 \mathrm{~g} \mathrm{animal}^{-1} \mathrm{día}^{-1} ; 4.45$ y 5.01, respectivamente), resultados que permiten sugerir la incorporación de hasta un $30 \%$ de estos recursos en dietas balanceadas para conejos de engorde. Quintero (1993), quien con el propósito de evaluar dos leguminosas Gliricidia sepium (Matarratón) y Cajanus cajan (gandúl) como fuentes de proteína con diferentes suplementos energéticos en la alimentación de conejos en crecimiento, ejecutó dos experimentos, reportando los mejores resultados con heno de matarratón para la ganancia de peso y la conversión alimenticia (18.7 $\mathrm{g}_{\text {animal }}{ }^{-1}$ día $^{-1}$ y 3.48; $20.7 \mathrm{~g}$ animal $^{-1}$ día $^{-1}$ y 3.68 , respectivamente).

Los menores consumos del matarratón tanto en la etapa de reproducción como la de producción, podrían estar asociados a la baja palatabilidad y a los contenidos de taninos existentes en esta leguminosa forrajera, relacionándose con lo expresado por Reed (1995), quien

Cuadro 3. Análisis económico de los tratamientos en la fase de engorde de conejos Neozelandeses

\begin{tabular}{|c|c|c|c|c|c|c|c|}
\hline \multirow{3}{*}{ Rubros } & \multicolumn{3}{|c|}{ Kudzú } & \multicolumn{3}{|c|}{ Matarratón } & \multirow{3}{*}{ Testigo } \\
\hline & \multicolumn{6}{|c|}{ Niveles de banano maduro (g) } & \\
\hline & 100 & 125 & 150 & 100 & 125 & 150 & \\
\hline Total egresos & 37.04 & 37.71 & 39.46 & 36.79 & 36.79 & 36.27 & 33.72 \\
\hline Total ingresos & 47.96 & 49.15 & 48.52 & 38.09 & 39.14 & 39.07 & 38.44 \\
\hline Beneficio neto & 10.92 & 11.44 & 9.06 & 1.30 & 2.35 & 2.80 & 4.72 \\
\hline Rentabilidad (\%) & 29.48 & 30.34 & 22.96 & 3.53 & 6.39 & 7.72 & 14.00 \\
\hline
\end{tabular}

indica que esta es una especie que contiene compuestos secundarios con propiedades antinutricionales, siendo uno de los metabolitos secundarios más importantes los taninos condensados; los cuales pueden tener efectos positivos o negativos en la digestibilidad de la proteína, los carbohidratos y la fibra del alimento. Además de estas sustancias también contienen pinitol, cumarinas, ácido melilótico, ácido ocumárico, glucósidos cianogénicos y nitrato (Aquiles et al., 1998).

\section{Conclusiones}

$\mathrm{L}$ a leguminosa de mayor consumo en la etapa de reproducción y producción fue el kudzú tropical (Pueraria phaseoloides (Roxb) Benth). El consumo de banano maduro se incrementa conforme aumenta su disponibilidad. Las leguminosas y los niveles de banano maduro no influenciaron sobre el peso de las hembras al parto, duración de la gestación, peso y tamaño de la camada al nacimiento. El peso y tamaño de la camada al destete, ganancia de peso y el rendimiento a la canal fueron superiores en los animales alimentados con kudzu tropical. La mayor rentabilidad en la etapa de engorde la presentó el tratamiento en base a kudzu tropical combinado 125 y $100 \mathrm{~g}$ de banano maduro animal $^{-1}$ día $^{-1}$.

\section{LiterATURA CITADA}

Aquiles, E., E. Moreno, A. Ojeda. 1998. Valor nutritivo de la Gliricidia sepium. Un árbol multipropósito. Fundación polar. Universidad Central de Venezuela. pp. 13-25.

Cheeke, P. R. 1992. Feeding systems for tropical rabbit production emphasizing root, tubers and bananas. FAO-Roots, tubers, plantain and bananas in animal 
feeding. p. 235-249

De Blas, C. 1989. Alimentación del conejo $2^{\mathrm{a}}$ ed. España, Mundi Prensa. pp. 34-62.

García, D., P. Lara, E. Magaña, E. Aguilar, J. Sangines. 2009. Parámetros reproductivos en conejas alimentadas con morera (Morus alba) ó tulipán (Hibiscus rosa-sinensis). Revista Verde (Mossoró - RN - Brasil), v.4, n. 3, p. 90-98.

Lebas, F., P. Coudert, H. de Rochambeau y R. Thébault. 1997. The Rabbit-Husbandry, Health and Production. FAO Animal Production and Health Series $\mathrm{N}^{\circ}$ 21. FAO. Roma Cambridge. pp: 255-272.

López, O., I. Montejo y L. Lamela. 2001. Evaluación de indicadores productivos en conejas mestizas con una dieta basada en forraje y pienso criollo. Estación Experimental de Pastos y Forrajes "Indio Hatuey". Matanzas, Cuba. 34(1):97-108.

Maertens, L. y M. Villamide. 1998. Feeding Systems for Intensive Production In: C. de Blas y J. Wiseman (eds.). The Nutrition of Rabbits. CABI Publishing. Cambridge. pp: 255-272.

Mbanya, J. N., B. N. Ndoping, J. M. Mafeni, D. W. Fomunyam. 2005. The effect of different protein sources and their combination on the performance of growing ISSN: 1021- rabbits in tropical conditions. Livestock Research for Rural Development 17(3). Art. No. 32. (on line). Researched 20 oct. 2010. Available in http://www.lrrd.org/lrrd17/3/mban 17032.htm
Nieves, D., O. Terán, M. Vivas, G. Arciniega, C. González, J. Ly. 2009. Comportamiento productivo de conejos alimentados con dietas basadas en follajes tropicales. Rev. Cient. (Maracaibo), 19(2):173-180. (en línea). Consultado 5 Sept. 2011. Disponible en: http://www.scielo.org.ve/scielo.php?Script=sciartt ext\&pid=S0798-22592009000200011 \&lng=es

Nouel, G., M. Espejo, R. Sánchez, P. Hevia, H. Alvarado, A. Brea, Y. Romero y G. Mejías. 2003. Consumo y digestibilidad de bloques nutricionales para conejos, compuestos por tres forrajeras del semiárido comparadas con soya perenne. Barquisimeto-Venezuela. Bioagro 15(1):1-10.

Quintero, V. 1993. Evaluación de leguminosas arbustivas en la alimentación de conejos. Departamento de Producción Animal, Universidad Nacional de Colombia, Facultad de Ciencias Agropecuarias. Palmira-Colombia. Livestock Research for Rural. 5(3).

Reed, J. D. 1995. Nutritional toxicology of tannins and related polyphenols in forage legumes. Journal Animal Science. 73:1516-1528.

Tuárez, J. 1977. Evaluación del rendimiento y valor nutritivo de gramíneas y leguminosas forrajera pertenecientes a la colección de la Estación Experimental, tesis Dr. en Ciencias Veterinarias. Universidad Técnica de Manabí. pp. 50. 